\title{
Rebuttal to reply by G. Rücker and G. Antes on Tass et al. "Counteracting tinnitus by acoustic coordinated reset neuromodulation", Restorative Neurology and Neuroscience Vol. 30(2), 2012
}

\author{
Peter A. Tass ${ }^{\mathrm{a}, \mathrm{b}, *}$, Ilya Adamchic ${ }^{\mathrm{a}}$, Hans-Joachim Freund ${ }^{\mathrm{a}}$, Tatjana von Stackelberg ${ }^{\mathrm{c}}$ \\ and Christian Hauptmann ${ }^{\mathrm{a}}$ \\ ${ }^{a}$ Research Center Jülich, Institute for Neuroscience and Medicine - Neuromodulation INM-7, Jülich, Germany \\ ${ }^{\mathrm{b}}$ Department of Stereotaxic and Functional Neurosurgery, University Hospital, Cologne, Germany \\ ${ }^{\mathrm{c}}$ Ear, Nose and Throat (ENT) Center, Meerbusch, Germany
}

We would like to thank Dr. Gerta Rücker and Prof. Gerd Antes for their interest in our article "Counteracting tinnitus by acoustic coordinated reset neuromodulation" and for their suggestions especially as the Cochrane collaboration has a long-standing track record for reviewing tinnitus therapies.

Specifically, they found very limited evidence for efficacy of commonly used tinnitus therapies (www.thecochranelibrary.com) such as tinnitus retraining therapy (TRT, Phillips \& McFarran 2010) and cognitive behavioral therapy (CBT, MartinezDevesa et al. 2010). Cochrane defines evidence as not limited to randomized controlled trials and metaanalyses but including the best available evidence at any one time encompassing all kinds of medical research, which can be also the result from basic

*Corresponding author: Peter A. Tass, Research Center Jülich, Institute for Neuroscience and Medicine - Neuromodulation INM7, Jülich, Germany. Tel.: +49 246161 2087; Fax: +49 246161 1880; E-mail: p.tass@fz-juelich.de. research or an expert consensus (see e.g. Sackett et al. 1996).

In the field of tinnitus, most of the trials are not performed blinded and often use a waiting list control instead of a true placebo group (MartinezDevesa et al. 2010). Patient numbers of 20 to 60 per study are a common size (Goebel and Hiller 1998, www.thecochranelibrary.com).

The RESET trial was conducted according to requirements of ICH-GCP and the German national legislation. With 63 patients using a randomized, blinded, placebo controlled setting, the trial shows quality features not often encountered in tinnitus research and appropriate for a first in man proof-ofconcept trial.

The concept was proven: we were able to show strong long-lasting effects of acoustic CR neuromodulation with reductions in loudness, annoyance and other tinnitus symptoms. The clinical effects were reflected in EEG changes towards a normal EEG spectrum in tinnitus relevant brain areas. Neither the 
placebo group nor the masker like group showed any lasting effects.

The results of the RESET trial support the robustness of acoustic CR neuromodulation with significant effects in the groups $\mathrm{G} 1$ and G3, receiving 4 to 6 hours CR therapy per day. Furthermore, the study showed that treatment effects did not correlate with tinnitus duration, tinnitus severity or patient's age. The protocol-defined analyses were complemented by analyses comprising the comparison of equally sized, matched subgroups and the pooled comparison between 'effective' and 'ineffective' stimulation groups. These additional analyses were requested by the peer reviewers and were carried out post-hoc. The results strengthen our conclusion that acoustic CR neuromodulation (G1 \& G3) had stronger long-lasting effects on tinnitus severity than the other treatments used in the trial.

The excellent response rate after the long-term extension $-75 \%$ responders and winners with a TQ reduction $\geq 6$ points - confirmed a high prevalence of a therapeutic effect at the individual-patient level. These $75 \%$ responders and winners experienced an average TQ reduction of $50 \%$. We would like to highlight, that from the very beginning we listed the relative reduction of the mean TQ value of all subjects in the article $(36 \%)$. Also the $25 \%$ non-responders and losers experienced a slight average TQ improvement. In accordance with other groups with a long-standing track record in the field of tinnitus (see Goebel et al. 2006; Landgrebe et al. 2012), we think that such a responder analysis based on minimal clinically meaningful changes is a useful tool for the interpretation of study results (Adamchic et al. 2012a, b).

We performed an additional analysis of covariance (ANCOVA) with treatment group as factor and respective baseline values as covariates as suggested by Dr. Gerta Rücker and Prof. Gert Antes. The results further support our statement that acoustic CR neuromodulation induced beneficial long-lasting effects. Despite the small group sizes, the direct comparison of change from baseline between the groups at 12 weeks revealed group differences with $p$-values $<0.05$ for the three parameters VAS annoyance $(p<0.05)$, VAS loudness $(p<0.05)$, and TQ $(p<0.0001)$ in the off state. No significant differences were found in the on state (VAS annoyance and VAS loudness). For example, this analysis showed that longer CR neuromodulation resulted in stronger therapeutic effects. In particular, differences $(p<0.05)$ where found comparing VAS loudness (off state) for groups G1 \& G3 (4-6h of CR neuromodulation per day) with G4 (only 1 hour of CR neuromodulation per day). Even stronger effects were obtained for TQ.

Further analysis revealed that acoustic CR neuromodulation actually reversed the pathological EEG characteristics (Adamchic et al., 2012c). CR therapy reduced cortical synchronization at low (delta and low theta) and high (beta, low and high gamma) frequencies and increased activity in the alpha band. These clinical and neurophysiological effects of CR neuromodulation are also supported by other experimental studies providing further evidence for the CR approach. For instance, acute desynchronizing effects (Neiman et al., 2007) and long-lasting desynchronizing after-effects (Tass et al., 2009, 2012b) of electrical CR were demonstrated in animal experiments. In fact, computational studies (Tass \& Popovych 2012; Popovych \& Tass 2012) show that CR neuromodulation can be realized by means of invasive (e.g., electrical) as well as noninvasive (e.g., sensory) stimulation modalities.

RESET is part of a long-term research program at the Research Center Jülich. It builds on a model based (Tass 1999) approach of reversing pathophysiological synchronization underlying tinnitus and other brain disorders characterized by abnormal synchronization. It was explored in computational models (Tass, 2003) as well as animal studies (Tass et al., 2009, 2012b).

The long-lasting reduction of subjective tinnitus loudness and reversed tinnitus-specific increased cortical synchronization clearly distinguishes acoustic CR neuromodulation from the currently widely used therapies. As formerly mentioned this is a proof of concept trial, but in contrast to other purely empirical therapies it is based on a well elaborated scientific concept that is widely accepted and experimentally supported. Our results demonstrate that acoustic CR neuromodulation has an effect on the postulated pathophysiological causes of tinnitus (Dohrmann et al., 2007; Kahlbrock and Weisz, 2008; Tass et al., 2012a; Weisz et al., 2005). In contrast, common therapies, e.g. TRT or CBT, aim at one of three response systems (behaviour, cognition and physiological reactivity) and are unlikely to have an effect on the pathophysiological causes of tinnitus (Martinez-Devesa et al., 2010; Phillips \& McFarran 2010).

The RESET study is part of a major clinical development program focusing on tinnitus treatment with CR neuromodulation. As a next step and based on the effects and the safety data obtained in the reported 
study, the statistically powered prospective multicenter, double blinded, placebo-controlled, randomized trial (RESET 2, NCT01541969) was started in September 2012. This study randomizes 100 patients with chronic tonal tinnitus either to placebo or acoustic CR neuromodulation as used in the G1 group of the RESET trial.

We hope to clarify most of the questions and would like to take this opportunity to invite Dr. Rücker and Prof. Antes to come to the Research Center Jülich for discussing the ongoing clinical studies as well as the future development program.

Yours faithfully, The authors

\section{References}

Adamchic, I., Langguth, B., Hauptmann, C. \& Tass, P.A. (2012a). Psychometric evaluation of Visual Analog Scale for the assessment of chronic tinnitus. Am J Audiol, 21, 215-225.

Adamchic, I., Tass, P.A., Langguth, B., Hauptmann, C., Koller, M., Schecklmann, M., Zeman, F. \& Landgrebe, M. (2012b). Linking the Tinnitus Questionnaire and the subjective Clinical Global Impression: Which differences are clinically important? Health Qual Life Outcomes, 10, 79.

Adamchic, I., Hauptmann, C. \& Tass, P.A. (2012c). Reversing pathological neuronal synchrony and tinnitus symptoms by acoustic CR neuromodulation in: https://ww4. aievolution.com/hbm1201/files/content/abstracts/98203/0214_ Adamchic.pdf(Poster presented at: The 18th Annual Meeting of the Organization for Human Brain Mapping. Beijing, China).

Dohrmann, K., Elbert, T., Schlee, W. \& Weisz, N. (2007). Tuning the tinnitus percept by modification of synchronous brain activity. Restor Neurol Neurosci, 25, 371-378.

Goebel, W. \& Hiller, W. (1998). Tinnitus-Fragebogen (TF), Ein Instrument zur Erfassung von Belastung und Schweregrad bei Tinnitus. Hogrefe Verlag.

Goebel, G., Kahl, M., Arnold, W. \& Fichter, M. (2006). 15-year prospective follow-up study of behavioral therapy in a large sample of in patients with chronic tinnitus. Acta Otolaryngol Suppl, 556, 70-79.

Kahlbrock, N. \& Weisz, N. (2008). Transient reduction of tinnitus intensity is marked by concomitant reductions of delta band power. BMC Biol, 16(6), 4.
Landgrebe, M., Azevedo, A., Baguley, D., Bauer, C., Cacace, A., Coelho, C., Dornhoffer, J., Figueiredo, R., Flor, H., Hajak, G., van de Heyning, P., Hiller, W., Khedr, E., Kleinjung, T., Koller, M., Lainez, J.M., Londero, A., Martin, W.H., Mennemeier, M., Piccirillo, J., De Ridder, D., Rupprecht, R., Searchfield, G., Vanneste, S., Zeman, F. \& Langguth, B. (2012). Methodological aspects of clinical trials in tinnitus: A proposal for an international standard. J Psychos Res, 73, 112-121.

Martinez-Devesa, P., Perera, R., Theodoulou, M. \& Waddell, A. (2010). Cognitive behavioural therapy for tinnitus. Cochrane Database Syst Rev, CD005233.

Neiman, A.B., Russell, D.F., Yakusheva, T.A., DiLullo, A. \& Tass, P.A. (2007). Response clustering in transient stochastic synchronization and desynchronization of coupled neuronal bursters. Phys Rev E Stat Nonlin Soft Matter Phys, E76, 021908.

Phillips, J.S. \& McFerran, D. (2010). Tinnitus Retraining Therapy (TRT) for tinnitus (Review). Cochrane Database Syst Rev, CD007330.

Popovych, O.V. \& Tass, P.A. (2012). Desynchronizing electrical and sensory coordinated reset neuromodulation. Front Hum Neurosci, 6, 58.

Sackett, D.L., Rosenberg, W.M.C., Gray, J.A.M., Haynes, R.B. \& Richardson, W.S. (1996). Evidence based medicine: What it is and what it isn't. $B M J, 312,71$.

Tass, P.A. (1999). Phase Resetting in Medicine and Biology: Stochastic Modelling and Data Analysis. Berlin: Springer.

Tass, P.A. (2003). A model of desynchronizing deep brain stimulation with a demand-controlled coordinated reset of neural subpopulations. Biol Cybern, 89, 81-88.

Tass, P., Silchenko, A., Hauptmann, C., Barnikol, U. \& Speckmann, E.J. (2009). Long-lasting desynchronization in rat hippocampal slice induced by coordinated reset stimulation. Phys Rev E Stat Nonlin Soft Matter Phys, 80, 011902.

Tass, P.A., Adamchic, I., Freund, H.J., von Stackelberg, T. \& Hauptmann, C. (2012a). Counteracting tinnitus by acoustic coordinated reset neuromodulation. Restor Neurol Neurosci, 30, 137-159.

Tass, P.A. \& Popovych, O.P. (2012). Unlearning tinnitus-related cerebral synchrony with acoustic coordinated reset stimulation - theoretical concept and modelling. Biol Cybern, 106, 27-36.

Tass, P.A., Qin, L., Hauptmann, C., Dovero, S., Bezard, E., Boraud, T. \& Meissner, W.G. (2012b). Coordinated reset has sustained after-effects in Parkinsonian monkeys. Ann Neurol, 72, 816820.

Weisz, N., Moratti, S., Meinzer, M., Dohrmann, K. \& Elbert, T. (2005). Tinnitus perception and distress is related to abnormal spontaneous brain activity as measured by magnetoencephalography. $P \operatorname{LoS} \operatorname{Med} 2$, e153. 